University of Nebraska - Lincoln

DigitalCommons@University of Nebraska - Lincoln

Faculty Publications from Nebraska Center for Research on Children, Youth, Families, and Schools
Children, Youth, Families \& Schools, Nebraska Center for Research on

\title{
$5-2009$
}

\section{Professional Development to Support Parent Engagement: A Case Study of Early Childhood Practitioners}

Jill R. Brown

Creighton University

Lisa L. Knoche

University of Nebraska-Lincoln, lknoche2@unl.edu

Carolyn P. Edwards

University of Nebraska - Lincoln, cedwards1@unl.edu

Susan M. Sheridan Dr.

University of Nebraska at Lincoln, ssheridan2@unl.edu

Follow this and additional works at: https://digitalcommons.unl.edu/cyfsfacpub

Part of the Pre-Elementary, Early Childhood, Kindergarten Teacher Education Commons

Brown, Jill R.; Knoche, Lisa L.; Edwards, Carolyn P.; and Sheridan, Susan M. Dr., "Professional Development to Support Parent Engagement: A Case Study of Early Childhood Practitioners" (2009). Faculty Publications from Nebraska Center for Research on Children, Youth, Families, and Schools. 14. https://digitalcommons.unl.edu/cyfsfacpub/14

This Article is brought to you for free and open access by the Children, Youth, Families \& Schools, Nebraska Center for Research on at DigitalCommons@University of Nebraska - Lincoln. It has been accepted for inclusion in Faculty Publications from Nebraska Center for Research on Children, Youth, Families, and Schools by an authorized administrator of DigitalCommons@University of Nebraska - Lincoln. 


\title{
Professional Development to Support Parent Engagement: A Case Study of Early Childhood Practitioners
}

\author{
Jill R. Brown \\ Creighton University
}

\section{Lisa L. Knoche, Carolyn P. Edwards, and Susan M. Sheridan}

Nebraska Center for Research on Children, Youth, Families, and Schools University of Nebraska-Lincoln

\author{
Corresponding author - Dr. Lisa L. Knoche, 238 Teachers College Hall, \\ University of Nebraska-Lincoln, Lincoln, NE 68588; email lknoche2@unl.edu
}

\begin{abstract}
Research Findings: This qualitative case study describes early childhood practitioners' (ECPs) perspectives on their professional development as part of a large federally funded school readiness intervention project as they experienced the processes of professional growth and change in learning skills related to promoting parental engagement in children's learning and development. A total of 28 ECPs participated in this study over 2 assessment periods across 2 academic years; 12 ECPs were interviewed twice, for a total of 40 interviews conducted and analyzed. Practitioners worked within the context of Early Head Start, Head Start, and Student Parent Programs in local high schools, all located in a midwestern state. The study intended to (a) discover practitioners' understanding of a parent engagement intervention, including their perspectives on the professional development and supports received; (b) assess how the parent engagement intervention was experienced by ECPs; and (c) discern how self-reported attitudes and behaviors of practitioners toward work with families changed as a function of the professional supports they received. Qualitative analyses of interview transcripts revealed 3 primary themes contributing to ECPs' experience with and understanding of the professional development model to support parent engagement: Self-Perceived Changes in Confidence and Competence in Enhancing Parental Engagement, Relationships as Supports for Change, and Practice: Time Pressure and Paperwork Woes.

Practice or Policy: Lessons learned and implications for the implementation of future professional development models are provided. Findings inform other
\end{abstract}


early childhood professional development efforts being implemented in the context of rigorous, research-based programming, particularly those intending to support parent engagement.

\section{Introduction}

In the field of early care and education, the need for high-quality programming to support the development of children and families is unquestioned. The current legislative landscape, with multistate support of pre$\mathrm{K}$ programs as well as federal initiatives such as Good Start, Grow Smart (Bush Administration, 2002), has prompted early childhood educators to demonstrate specific skill development in children they serve (Harbin, Rous, \& McLean, 2005; No Child Left Behind Act, 2002). In this heightened climate of accountability, professional development opportunities offered to practitioners are the primary means through which administrators of programs and service agencies attempt to achieve high-quality programming and, ultimately, contribute to positive child and family outcomes.

Most early childhood professional development studies to date have focused on events occurring within early childhood classrooms. These studies have focused on topics such as enhancing program quality in classrooms (Fontaine, Torre, Grafwallner, \& Underhill, 2006; Howes, James, \& Ritchie, 2003), facilitating specific child skills (Dickinson \& Brady, 2006; Ginsburg et al., 2006; Jackson et al., 2006), and implementing curriculum with fidelity to promote child outcomes (Justice, Mashburn, Hamre, \& Pianta, 2008). School readiness, however, can be conceptualized ecologically and can extend beyond the walls of the early childhood classroom to include children's and teachers' relationships with parents and other caregivers (Mashburn \& Pianta, 2006; Snow, 2006). The quality of relationships that children experience early on with parents sets the stage for later competence in preschool and school settings (Bornstein \& Tamis-LeMonda, 1989; de Ruiter \& van IJzendoorn, 1993; Raver \& Knitzer, 2002; Shonkhoff \& Phillips, 2000; Thompson, 2002). Parental engagement specifically has been linked to school readiness skills, including young children's academic performance and achievement (Foster, Lambert, Abbott-Shim, McCarty, \& Franze, 2005; Hill, 2001; McWayne, Hampton, Fantuzzo, Cohen, \& Sekino, 2004; Weigel, Martin, \& Bennett, 2006), their prosocial behaviors (McWayne et al., 2004), and positive approaches to learning (Turner \& Burke, 2003). Given these important associations, professional development efforts must also extend beyond the early childhood classroom.

Professional development studies within the early childhood field conducted heretofore have investigated the outcomes of both teacher professional development and parent engagement efforts. However, little is known about the beliefs and experiences of practitioners as they work to develop skills to engage parents and build collaborative partnerships with 
families. Furthermore, limited data are available on the professional development that practitioners receive to help facilitate change in parental engagement. Empirical study is needed to understand the perspectives of professionals as they attempt to incorporate new interventions to support family engagement in child learning into their professional work with families. The current study investigated a professional development effort focused on practitioner's abilities and skills in promoting parent-child engagement and complements past studies focused on the implementation of early childhood classroom curricula.

The purpose of this study was to understand early childhood practitioners' (ECPs) perspectives on their professional development process and perceptions of how children, families, and they themselves were changed as a result of being part of a large, federally funded school readiness intervention project designed to enhance parental engagement (Sheridan \& Edwards, 2003). The intervention was intended to improve child outcomes by promoting the confidence and competence of parents in their role of preparing their children for school. To be successful, the intervention also needed to promote (in a parallel way) the confidence and competence of the ECPs who were working directly with parents and children, and this was the focus of professional development efforts. The study provides data relevant to understanding practitioners' responses to the challenges and rewards of learning new skill sets for working collaboratively with parents on behalf of children's learning and development. As such, the study offers needed insights into the specific issue of professional development focused on promoting parent engagement in early care and education as a key area of content.

\section{The Research Context}

The Getting Ready project is a large federally funded school readiness research project (Sheridan \& Edwards, 2003) that integrates content related to family engagement in the context of existing early childhood programming. In the Getting Ready intervention model (Sheridan, Marvin, Knoche, \& Edwards, 2008), practitioners provide early intervention and education services for parents and children aged birth to 5 years through a prevention lens that (a) guides parents to engage in warm and responsive interactions, support their children's autonomy, and participate in children's learning; and (b) supports parents and ECPs in collaborative interactions to support children's learning and development in home- and center-based settings.

The Getting Ready parent engagement intervention. The Getting Ready intervention integrates triadic (McCollum \& Yates, 1994) and collaborative (conjoint) consultation (Sheridan \& Kratochwill, 1992, 2008) models in the delivery of family-centered services. The integration of these models results in triadic/collaborative planning strategies as the basis of the Getting Ready intervention. Specifically, triadic strategies are integrated with collaborative planning practices to mutually support parent-child and family- 
school relationships. The triadic model is a research-based intervention (Girolametto, Verbey, \& Tannock, 1994; Mahoney \& Powell, 1988; McCollum, Gooler, Yates, \& Appl, 2001). Its aim is to facilitate success in preschool and the eventual transition to kindergarten by strengthening parents' warmth and sensitivity and support of their child's autonomy and learning within the normal framework of families' communication and guidance styles in everyday parent-child interactions and daily routines. Collaborative planning strategies are those wherein parents and ECPs jointly plan for a child's learning and development. These strategies support the bidirectional sharing of information and experiences, joint attention to child needs, mutual goal setting, and shared decision making (Sheridan \& Kratochwill, 2008). Through focused collaborative exchanges, parents and ECPs are engaged as partners to co-construct the learning process in ways that are important and meaningful for all participants (Christenson \& Sheridan, 2001).

The Getting Ready intervention is being implemented in the natural context of community early childhood programs (Early Head Start, Head Start and high school Student Parent programs) via ECPs. Rather than representing an "add on" to current services, it is integrated with and strengthens the ongoing means for intervening with families. ECPs in home-based Early Head Start programs (serving children aged birth to 3 years) are taught to utilize triadic and collaborative planning during weekly home visits as well as during monthly group activities ("socializations") held with families. Practitioners in the center-based high school Student Parent programs (serving children aged birth to 3 years) are taught to use these strategies as they engage daily with families and have twice-monthly individual meetings with parents and children. Finally, professionals in preschool center-based Head Start programs (serving children aged 3-5 years) utilize the strategies during bimonthly home visits, parent-teacher conferences, and group activities (socializations) held at the school or at a special site such as the municipal children's museum or zoo.

Professional development to support parent engagement. The primary purpose of professional development in Getting Ready is to support ECPs' developing competence and confidence in their interactions with parents so as to support parents' own competence and confidence in their interactions with their own children. The model of professional development is relationship based. The primary components of professional development in the Getting Ready model are a training institute, individual coaching, and group coaching.

Prior to beginning to deliver the intervention, the ECPs were introduced to triadic/collaborative planning (T/CP) strategies in an initial, 2-day institute devoted to T/CP strategies and their use in home visits, socializations, and other interactions with families. Practitioners then received coaching twice per month from a project coach to support their use of the strategies. The purpose of Getting Ready coaching was to support the initial training 
and move practitioners toward use of the triadic/collaborative strategies in their practice with families. One session each month was individualized, whereas a second session took place in a group format with three to five ECPs from the same program. Individual sessions were $1 \mathrm{hr}$ in length; group sessions were $1 \frac{1 / 2}{2}$ to 2 hours. Coaches were three females with master's degrees in a human services field, and extensive experience in parent consultation and early childhood intervention and education.

The ECPs' strengths and needs informed the content of coaching sessions, as coaches tailored sessions for each individual. Each session had a primary focus on a single triadic/collaborative strategy. The project coach provided support, asked questions, helped create professional goals, and discussed strengths and challenges. Group sessions provided the opportunity for peers to share ideas for interventions, develop cohesive relationships around specific professional goals, and learn with and from one another. The coaching sessions were driven by the Coaching Families and Colleagues model (Hanft, Rush, \& Shelden, 2004). Each coaching session consisted of four phases, as described in Table 1.

Coaching sessions were augmented with in vivo observations by the project coach of individual ECPs during interactions with families. On select home visits and socializations, the coach accompanied the early childhood practitioner and made a video recording of the session. Selected segments of parent-ECP interaction were edited and used in coaching sessions, first in individual and then in group sessions. Recorded episodes of positive interactions were shown and discussed by the project coach with ECPs to reinforce desired behaviors and to extend self-awareness about the signif-

Table 1. Components of Professional Development in the Getting Ready Model

\begin{tabular}{ll}
\hline Component & Focus \\
\hline $\begin{array}{l}\text { Individual and Group } \\
\text { Coaching }\end{array}$ & $\begin{array}{c}\text { Opening (10\% of session) } \\
\text { Phase 1: Initiation - Clarify purposes, desired outcomes, and } \\
\text { agendas for coaching. } \\
\text { Main Agenda (80\% of session) } \\
\text { Phase 2: Observation and Action - Address teachers' and aides' } \\
\text { use of intervention strategies in classrooms and home vis- } \\
\text { its. Provide feedback, role-play, and model when appro- } \\
\text { priate. Use short activities, self-monitoring, videos, and } \\
\text { self-reflections as appropriate. } \\
\text { Phase 3: Reflection - Use reflective questioning and examples } \\
\text { from professionals' work with families; mutual dialogue; } \\
\text { and confirmation of strengths, challenges, and personal } \\
\text { goals to guide the discussion and develop professional } \\
\text { strengths in the use of triadic/collaborative strategies. } \\
\text { Closing (10\% of session) } \\
\text { Phase 4: Evaluation - Plan early childhood practitioners' use } \\
\text { of triadic/collaborative strategies between coaching } \\
\text { sessions. }\end{array}$ \\
\hline
\end{tabular}


icance of those behaviors. This "self-as-a-model" cognitive social-learning technique motivates self-coping skills in all practitioners, especially those who view themselves as being inadequate (Dowrick, 1994; Hosford, 1980). Use of video protocols was intended to enhance coaching by allowing participants to become more aware of their actions, analyze critical events, ascertain strengths and weaknesses, and establish personal goals.

Preliminary studies. Preliminary studies conducted by the research team have indicated that the Getting Ready parent engagement intervention was implemented with fidelity and resulted in observable changes in ECP behavior. First, a quantitative fidelity analysis using observational home visit data indicated that the performance of ECPs in the treatment group differed from that of participants in the control group in several ways. Relative to control group participants, ECPs in the treatment group effectively initiated parental interest and engagement at a significantly higher level, and used key Getting Ready strategies at a significantly greater rate. Similarly, parents and children were observed interacting with each other, and parents were rated as demonstrating significantly greater interest in and engagement with their child during home visits for treatment group participants as compared to control participants (Knoche, Sheridan, Edwards, \& Osborn, under review). Likewise, a qualitative study of parent communication documents and home visit reports (Sheridan, Knoche, Edwards, Bovaird, \& Kupzyk, in press) provided strong evidence of treatment teachers' spontaneous use of intervention strategies of collaborative planning and problem solving with parents relative to control teachers. Specifically, generalization of teachers' efforts to strengthen home-school collaboration, form relationships with parents, and spotlight and acknowledge child and parent competence was evidenced in teachers' communication records with families (i.e., home visit reports, newsletters).

These studies indicate that ongoing professional development with treatment participants in the parent engagement intervention can result in behavioral change in interactions and communications with families. Taken together with findings indicating that the parent engagement intervention was successful in improving child social-emotional outcomes (Sheridan, Knoche, Edwards, Bovaird, \& Kupzyk, in press) and parent engagement behaviors (Cline, Knoche, Edwards, Sheridan, \& Martinez, 2009), we have empirical evidence of the effects of the professional development activities. However, perceptions regarding the change process as well as the influence of change on professionals' perspectives have not been investigated. In an effort to understand the professional development process in a deeper way from the perspective of consumers, a study investigating the ECPs' own perceptions of the change process is warranted and. will contribute to our understanding of effective professional development approaches to support parent engagement and parent-child interactions. 
In an effort to contribute to the literature on professional development designed to enhance parent-child relationships, the research questions for this study are: (a) W hat is practitioners' understanding of a parent engagement intervention, including their perspectives on the professional development and supports received? (b) How was the parent engagement intervention experienced by the ECPs? and (c) How did self-reported attitudes and behaviors of practitioners toward work with families change as a function of the professional supports they received? Qualitative methods were used to glean personally rich information on practitioners' experiences and perspectives.

\section{Methods}

In general, qualitative approaches to research are based on a holistic worldview stressing that (a) reality is based upon perceptions that are different for each person and change over time, (b) there is not one single reality, and (c) what we know has meaning within a given context. As such, findings are not causal. It is from this worldview that we derived our research questions and chose our method of analysis. A qualitative case study design was used, defined as the in-depth study of a phenomenon bound in space and time (Stake, 1995). The Getting Ready project provided the context for looking closely at the professional and personal experiences of ECPs as they learned to implement a new intervention aimed at increasing parental engagement and children's school readiness. Because the intervention was organized on the premise of trusting and collaborative relationships, we believed it was imperative to inquire about the subjective experience of participants (e.g., what ECPs perceived to be the purpose of the intervention and the changes they were being asked to make in their behavior with parents, whether the training and support they received was adequate and helpful, if they felt they were changed by the experience of the parent engagement intervention). The case study method was chosen not only to provide formative and summative data on the rich and complex process of professional development, but also as a way for the researchers to stay true to the commitment of treating the ECPs as collaborators with the research team and to assess and respond to the needs of the research participants.

We used the three components delineated by Stake (1995) and Yin (1994) for conducting the case study. First, a complex description of the case being studied is presented. This includes the theoretical model behind the project, the specific components of the project, and a detailed description of the settings where the project was carried out. Second, we used thematic analysis to look for patterns in the data. Categorical aggregation of the data was used to find collections of instances where meaning was revealed. This is presented through themes using actual quotes from the participants as evidence. In this way, we establish patterns that emerged from ECPs' statements and look for correspondence between two or more cate- 
gories. Third, the interpretations and assumptions of the researchers are explored through naturalistic generalization. This occurs as we reflect on our findings, make cautious generalizations based on what we have learned from the case, and speculate about how others may apply the findings to different cases or populations.

\section{Setting and Participants}

Three different sites in a midwestern state served as settings for this study. The sites served children and families within the context of Early Head Start (EHS), Head Start (HS), and Student Parent Programs (SPPs) in local high schools.

A total of 28 individuals participated in this study over two assessment periods ("rounds"): 12 ECPs were interviewed during both Rounds 1 and 2; nine were interviewed only during Round 1, and seven were interviewed only during Round 2 (see Table 2). For Round 1, all 25 ECPs who were involved in the experimental condition of the Getting Ready project were contacted and invited to participate, and 21 agreed. For

Table 2. Number of Interviews and Participants Across Time and Categories of Practitioners

\begin{tabular}{|c|c|c|c|c|}
\hline \multirow[b]{2}{*}{ Variable } & \multirow{2}{*}{$\begin{array}{l}\text { Early Head } \\
\text { Start }\end{array}$} & \multicolumn{3}{|c|}{ Student Parent } \\
\hline & & Head Start & Program & Total \\
\hline \multicolumn{5}{|l|}{ Sample size per interview round $(n)$} \\
\hline Round 1 & 7 & 7 & 7 & $21^{\mathrm{ab}}$ \\
\hline a. Round 1 not returning for Round 2 & 2 & 3 & 4 & 9 \\
\hline b. Round 1 returning for Round 2 & 5 & 4 & 3 & $12^{\mathrm{b}}$ \\
\hline c. New participants added at Round 2 & 3 & 1 & 3 & $7^{\mathrm{ab}}$ \\
\hline Round $2(b+c)$ & 8 & 5 & 6 & 19 \\
\hline Total sample across rounds $(n)$ & 10 & 8 & 10 & 28 \\
\hline \multicolumn{5}{|l|}{ Demographic characteristics } \\
\hline Age ( $M$ years) & 35 & 44 & 40 & \\
\hline Early childhood experience ( $M$ years) & 4 & 13 & 14 & \\
\hline Time with current agency ( $M$ years) & 2 & 5 & 6 & \\
\hline \multicolumn{5}{|l|}{ Ethnicity (\%) } \\
\hline White & 80 & 100 & 100 & \\
\hline Hispanic & 20 & 0 & 0 & \\
\hline \multicolumn{5}{|l|}{ Education (\%) } \\
\hline$<2$-year degree & 21 & 0 & 17 & 13 \\
\hline 2-year degree & 58 & 0 & 33 & 30 \\
\hline 4-year degree or higher & 21 & 100 & 50 & 57 \\
\hline
\end{tabular}

a. A total of 28 individuals participated in the study; 21 were interviewed at Round 1 , and 7 new participants were added in Round 2.

b. A total of 40 interviews were conducted: Round 1 (21 early childhood practitioners [ECPs]) + Round 2 (12 returning ECPs and 7 new ECPs). 
Round 2, 12 ECPs were reinterviewed, but the remaining nine from Round 1 were no longer employed by their respective agencies. Staff turnover is common in early childhood programming (Bellm, Burton, Whitebook, Broatch, \& Young, 2002) and was characteristic of the Getting Ready project as well. To widen the sample and add further information regarding first-year experiences in the project, seven new participants (all new hires within their respective agencies/programs) were added to the sample for Round 2.

Early childhood practitioners were $100 \%$ female and were, on average, 39.6 years old. Ninety-three percent identified as White/Caucasian, and 7\% identified as Hispanic. Thirteen percent had less than a 2-year degree, 30\% had an associate's degree, and 57\% had a bachelor's degree. Table 2 displays the demographic characteristics of the sample by site.

Early Head Start. A total of 10 EHS practitioners were interviewed across the two rounds of interviews. The EHS practitioners were employed by two different community service agencies in two rural counties. One agency was located on a community college campus; the other was housed within a renovated retail building. EHS practitioners provided weekly home visit services to families focused on child development and parenting skills and held monthly family group socialization activities. Each practitioner had a caseload of 10 to 15 families and offered services prenatally through age 3 .

Head Start. Eight HS practitioners participated in the case study. The HS practitioners worked in center-based preschool classrooms located within elementary schools in a mid-size, midwestern community. Classrooms were in session during the academic year, 4 or 5 days each week, for $4 \mathrm{hr}$ each day. Lead teachers in the HS setting interacted with families during child drop-off and pick-up, during regularly scheduled family socialization activities at the school and in the community, and during home visits that occurred an average of five times each academic year. All HS practitioners were certified teachers with early childhood teaching endorsements.

Student Parent Program. A total of 10 individuals working within SPPs participated in the case study. Practitioners in the SPPs worked within child care centers located inside two of the secondary schools within a midsize, midwestern community. One of the programs was located in a large, traditional high school setting, and the other was housed within a smaller alternative high school. Each classroom had up to eight children with two staff members. Children in the program ranged in age from 2 weeks to 3 years and were separated into infant and toddler classrooms. 


\section{Procedures}

Semistructured qualitative interviews were conducted by the first author, who had no background experience with either the aims or methods of the Getting Ready project but who had extensive experience with interviewing and qualitative methodology through participation and leadership on other projects at the academic institution. Interviews were conducted in two distinct phases (or rounds) separated in time by approximately 1 year. Round 1 interviews occurred during the second year of the study; Round 2 interviews occurred during the third year of the study. Coaching had taken place in the year prior to the Round 1 interviews and in the year prior to the Round 2 interviews. Participants had been involved in coaching for up to 3 years by the point of the Round 2 interviews. A total of 40 interviews were conducted (21 in Round 1 and 19 in Round 2; see Table 2).

Each interview took approximately 30 to $90 \mathrm{~min}$, depending on the responses of participants. Interviews were conducted at the location of the participant's choice, usually her school or workplace for convenience. All interviews were audiotaped with the consent of the participant. The interview protocol is provided in Table 3. The questions in a qualitative study are generated by narrowing the central question and subquestions, bounded on the front by questions to develop rapport and at the end by questions about construct and ideas that may have been overlooked by the

Table 3. Protocol for Interviews with Early Childhood Practitioners

1. Think about your role as an early childhood practitioner over the past 3 years ... think about today. What is similar about your experience? How is your experience different?

2. I am not entirely familiar with the project, so forgive me, but would you describe the project? What is it about?

3. What has the Getting Ready project meant for you?

4. As the Getting Ready project has been implemented in your setting, what, if any, has been the effect on children in your classroom? Effect on families from your classroom? Effect on your program?

5. What kinds of support do you receive in the project?

a. Describe the professional support, and your reaction to the support, of workshops

b. coaching, and

c. Conjoint behavior consultation (CBC) consultants in classroom.

6. What are some highlights based on the support you have experienced?

7. Do you have any additional thoughts you would like to share on the Getting Ready project, content, and/or process?

Additional Questions Added for Round 2

1. What have you learned, if anything, through involvement that you will carry into next year? What kinds of behaviors and practices are "transportable" from the project?

2. Do you have any advice as we continue working with new as well as experienced early childhood practitioners? 
researcher (Creswell, 2003). Interview questions were developed by the research team to (a) tap into the experience of ECPs learning a new parent engagement intervention, (b) explore specific aspects of the intervention, and (c) serve as a formative evaluation of professional development. The protocol was designed to elicit the perspectives, opinions, and experiences of ECPs (Katz, 1992, 1995) that are informative for an audience interested in professional development to support parent engagement. In addition to answering the interview questions that composed the protocol, participants were encouraged by the interviewer through individualized followup prompts to expand on other areas of the project that they believed were important. This was used to inform the research team as active collaborators with the participants as well as to gather data on ECPs' experiences with aspects of the parent engagement intervention.

\section{Coding and Data Analysis}

Data analysis occurred over three phases. Audiotaped responses to the semistructured interview questions were transcribed by trained research assistants and analyzed for themes following a qualitative case study approach (Creswell, 2003; Stake, 1995). Qualitative data analysis builds codes, categories, and themes from the bottom up by organizing data from participants' responses into increasingly more abstract units in an inductive process (Creswell, 2003).

Phase 1: Initial coding. Graduate and undergraduate research assistants were trained by the first author. Initial analysis of transcripts was conducted by the first author, one graduate research assistant, and one undergraduate research assistant. First, all interviews were read by the entire research team, including the principal investigators, project director, and interviewer. Notes were taken by each individual regarding overall meaning found in the ECP responses. The first author and an undergraduate research assistant then worked independently to locate relevant segments of dialogue from the interview transcripts that described the practitioners' specific perceptions and experiences. Strauss and Corbin (1990) described this process as "fracturing the data," and it allows one to identify categories, their properties, and their dimensional locations. These segments of transcripts were then categorized using researcher-generated codes that captured their meaning based on the language of the participants. Sixtyfive initial codes were generated.

Phase 2: Theme generation. The second phase of transcript analysis was the generation of themes. The first author and an undergraduate researcher grouped the 65 initial codes into meaningful themes based on common experiences and words of participants. In an inductive process, single codes were clustered together by making connections between interrelated groups of codes and overarching meanings. For example, the in- 
dividual codes of "personal transformation," "internalization of the mission," and "looking forward" were grouped under a larger category of "sustainability." Ten content themes emerged from the analysis of the initial 65 codes.

Phase 3: Theme validation and disconfirmation. The final phase of transcript analysis was theme validation/disconfirmation. This involved an integrative process with two researchers reviewing the 10 content themes identified during Phase 2, generating the overarching core themes by relating content themes to one another based on meaning, and validating those relationships by searching for confirmation and disconfirmation (Strauss \& Corbin, 1990). The research team did this by returning to the 40 interview transcripts and identifying examples that confirmed and disconfirmed the 10 content themes. In reviewing the transcripts, not all of the content themes were evident across all participant interviews. The researchers discussed discrepancies between the transcripts and the 10 content themes and identified ways to collapse the 10 content themes into meaningful composite units, or overarching themes. To be retained as an overarching theme, the theme had to be centrally related to the other themes and mentioned by more than $25 \%$ of all participants. A consensus of the research team was reached and 6 themes from the original 10 were agreed upon. For the sake of parsimony, and in the interest of the journal's readership, these six themes were later reduced to three by consolidating and/or eliminating findings outside the scope of this article (i.e., ECPs' perceptions of their professional development). The consolidated themes were scope of professional development, perceived impact on children and families, teacher's explanation of the project, sustaining the Getting Ready mission, hope, and suggestions for improvement.

We encountered varying opinions among the ECPs in relation to some of the themes. For example, although over $25 \%$ of ECPs believed that coaching was a positive experience, not everyone agreed. To ensure validity and to honor our commitment to describing the breadth of experiences of practitioners, negative case analysis (disconfirmation) is presented in these instances to demonstrate the full range of the diversity of responses.

Interrater reliability among coders was calculated for 15\% of the transcripts generated in the first round of interviews (Cohen, 1960). Kappa was computed by considering exact agreement on a general code corrected for chance; Cohen's Kappa equaled .78. Codes and themes were explored across sites (EHS, HS, and SPPs) and across time (Rounds 1 and 2) to investigate differences.

\section{Themes}

Thematic analysis was conducted to uncover participants' experiences and understandings of the Getting Ready intervention and profes- 
sional development model. Specifically, the analysis allowed the researchers to (a) discover individual practitioners' understanding of the parent engagement intervention, including the professional development and supports received; (b) assess how the parent engagement intervention affected the behavior of the practitioners from the perspective of the ECPs; and (c) discern how self-reported attitudes and behaviors of practitioners toward work with families changed as a function of the professional supports they received. The analysis revealed three major themes: (a) Self-Perceived Changes in Confidence and Competence in Enhancing Parental Engagement, (b) Relationships as Supports for Change, and (c) Practice: Time Pressure and Paperwork Woes. Themes are presented below by using both description and exemplar interview segments.

\section{Self-Perceived Changes in Confidence and Competence in Enhancing Pa- rental Engagement}

The effects of the Getting Ready intervention on ECPs included professional change in practices and beliefs. One of the most pronounced themes from the interviews was the "spark" that the Getting Ready project brought to experienced as well as novice ECPs. One HS teacher who had taught for 25 years described her experience with the project: "I think it has improved my teaching. I think it has helped me get out of a rut. I feel I always come back to school and go home feeling more confident." Several ECPs used the phrase "confidence and competence" that the Getting Ready intervention endorses. One EHS practitioner said, "I'm more confident in myself about going into the homes and stuff and talking to the families. I do have to say the Getting Ready project and the coaches have been really helpful in that part." Other ECPs felt that the increase in their confidence was one of the most important aspects of the Getting Ready project. An EHS teacher said,

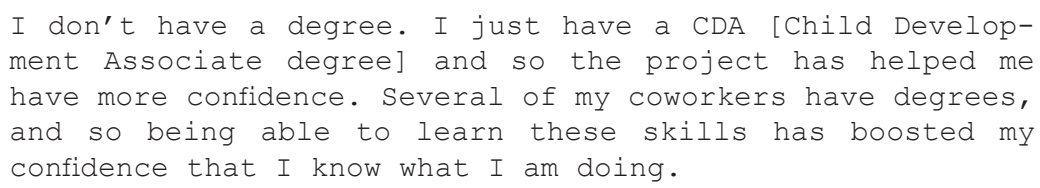

ECPs also described how their own professional practices had evolved. These participants appeared to have developed professional goals around strengthening the home-school connection, improving parent-teacher communication, and establishing a partnership with parents. One teacher poignantly said, "It doesn't have to just be teacher-child or parent-child but all of them together." Another said,

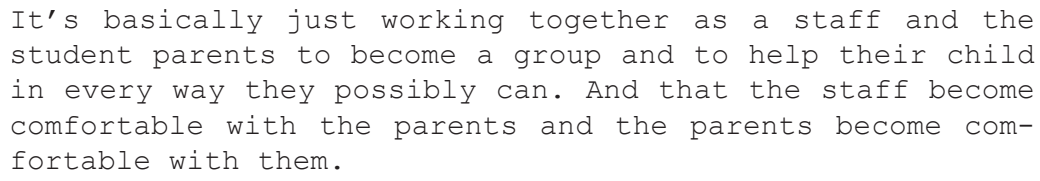


ECPs occasionally spoke of the good fit between the intervention and their own intentions. One ECP commented, "We weren't as consistent, but with the Getting Ready project, it became more of a consistency." Another spoke in detail about her belief in affirming parents and said that she understood the process better through involvement in the parent engagement intervention: "It's different than giving a compliment. It's ... more of, hey, I observed this; this is what I recognize. And it's pointing out the positives, and it's kind of putting it in their court."

Professionally, communication skills were enhanced between ECPs and parents as well as between ECPs themselves. A veteran HS teacher explained how her communication skills had changed after 1 year of the project:

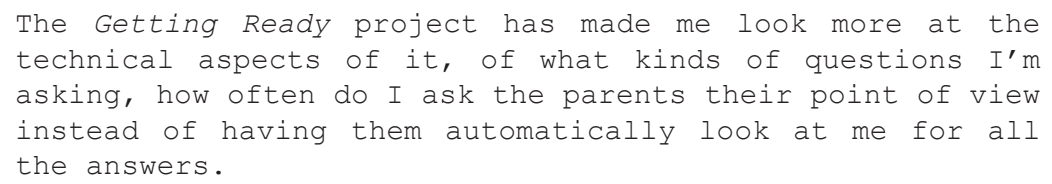

The focus of the parent engagement intervention-that of establishing a triadic relationship between the practitioner, parent, and child - had a transformative effect on the ECPs. The EHS practitioners more than the other groups endorsed this idea consistently. All ECPs thought their skills at engaging parents in the home visits had improved through their involvement in the Getting Ready project. For example, an EHS teacher described, "I learned a lot of things, like teaching parents how to be a better parent." This was accomplished by ECPs stepping back and giving some responsibility to the parents:

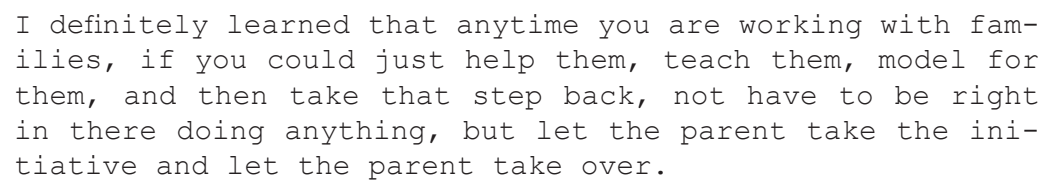

By engaging in triadic interactions (focusing on parent-child interactions within the parent-child-professional triad), ECPs developed skills to observe the impact of the intervention on families and children. Specifically, practitioners learned how to observe parents as they interacted with their children, and in turn how to help parents become better observers of their own children. The ability to step back and observe the families observing their children was noted by teachers as a change that the parent engagement intervention helped them foster. One HS teacher described how she sat back during a home visit and really watched the family:

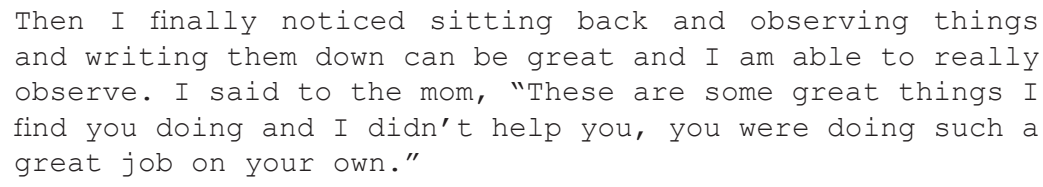


ECPs appreciated being made conscious of their efforts. Many described that they already had beliefs about education that were consistent with the goals of the Getting Ready intervention, but their involvement in the project and the support of coaches provided a needed boost to transform them as educational professionals. An HS teacher commented, "I am doing things with a little more purpose and emphasis relative to the project and just doing those things across the board." Another HS teacher commented,

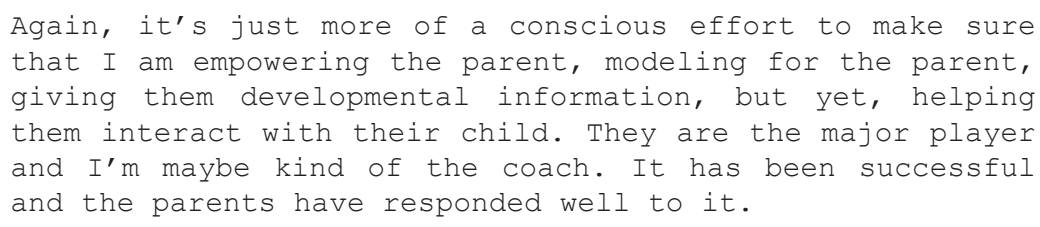

In addition to transformations and change in the professional realm, $12(30 \%)$ of the ECPs took things away from the intervention that changed them personally. ECPs appeared to internalize fundamental aspects of the parent engagement intervention and were able to apply them to their professional lives as well as to their lives away from job. One HS teacher explained that the project had "changed the entire way I do things, even in my personal life." Another explained, "I have several children, six children, so working with the Getting Ready project has changed my opinion of how I deal at home with my husband and children." She went on to describe how she incorporated skills from work into her family life by saying, "So, we are making cookies, we do the math; we stir, motor skills; and then I ask them what they think is going to happen."

Six ECPs spoke of their own internalization of the Getting Ready mission. One classroom teacher reflected about her evolution in thinking over her 3 years of involvement and attributed it to the Getting Ready intervention:

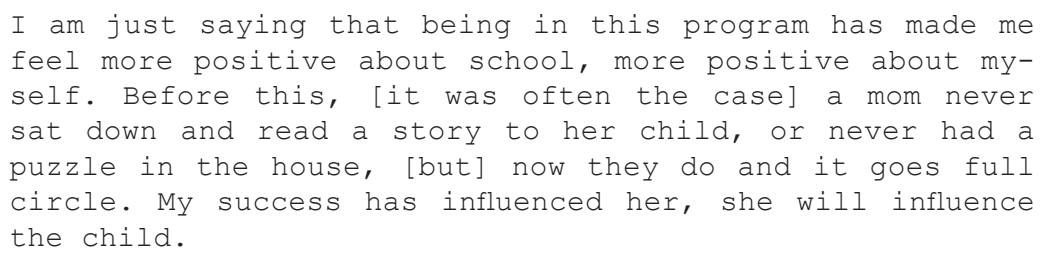

Another teacher with 31/2 years of experience adopted many of the Getting Ready philosophical tenets and explained that

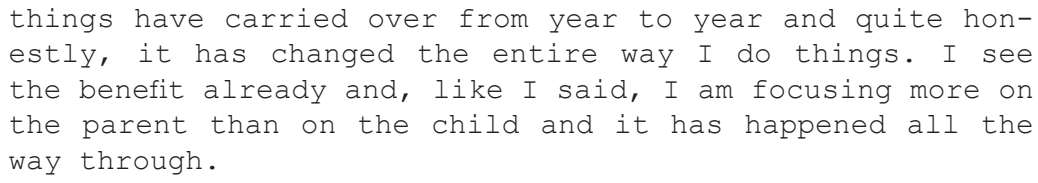


Through ECPs' involvement in the Getting Ready intervention and professional development, both their professional experiences and personal outlooks were affected. In general, practitioners reported positive shifts in their perspectives that reportedly enhanced their professional abilities and beliefs. Furthermore, practitioners experienced change beyond the classroom or agency setting; they reported experiences that indicated an internalized belief system and a desire to sustain the project that was affected by participation in the parent engagement intervention.

\section{Relationships as Supports for Change: Coaches and ECPs}

An important part of the parent engagement professional development was the relationship that formed between practitioners and coaches. Both the intensity and personalization of the coaching relationship were important components of professional development as practitioners learned new parental engagement strategies. One aspect of the evolving relationship between ECPs and coaches allowed the ECPs to watch a videotape of themselves on a home visit and critique it with their coach from the Getting Ready project. This highly personal experience allowed several ECPs to recognize the transformative aspects of triadic collaboration. One EHS teacher described,

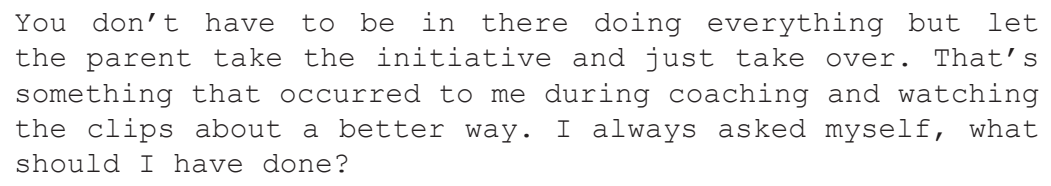

Trust was built between the ECPs and the coaches as the coaches delivered and the ECPs received constructive feedback. After a home visit, one ECP recalled, "I had a million questions so I called my coach. I know she is there for me." Coaches acted as model for giving feedback and specific praise. Five participants believed their experience with coaching helped them respond better with their families. One HS teacher expounded on this process:

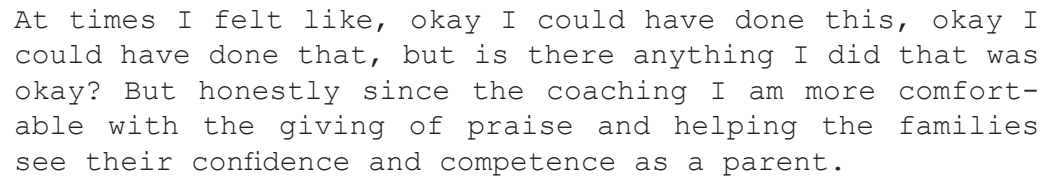

The opportunity that the group coaching created for ECPs to meet and talk with one another about all aspects of their jobs also played an important role in ECPs' professional development. Group coaching occurred for ECPs once per month. One HS teacher explained, 


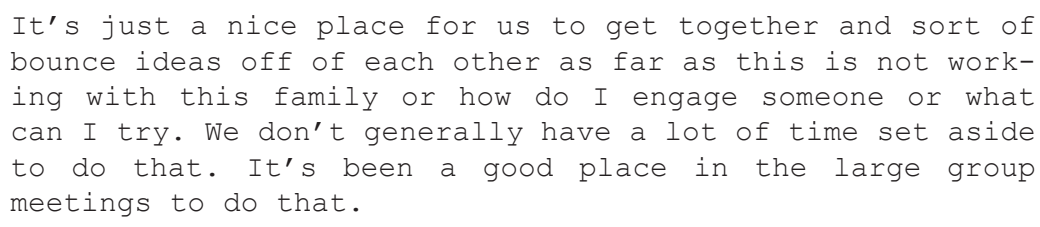

An environment of open communication and acceptance between Getting Ready staff and ECPs evolved from Round 1 to Round 2,1 and this appeared to have a significant effect on ECPs. In Round 1 the group coaching at times caused conflict between the ECPs. Nineteen (48\%) ECPs described their experience with the individual and group coaching as something that could be improved upon. Some ECPs were uncomfortable and expressed dissatisfaction with the personality and style of one of the coaches. Others reported that the group coaching and the individual coaching overlapped in content; they thought the time spent in coaching could have been more productive. One EHS practitioner explained,

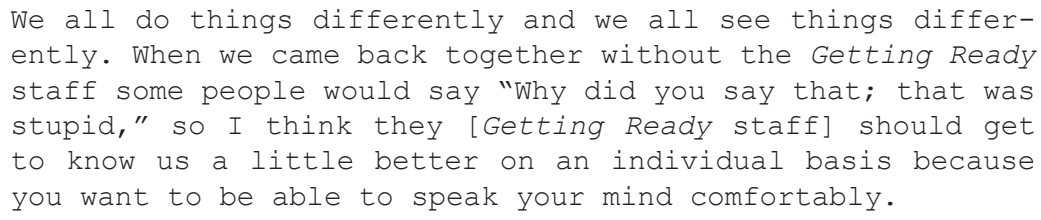

Personality conflicts were also experienced between some of the ECPs and their coaches. One ECP reported, "One particular coach has really rubbed me the wrong way and I have had a hard time even participating and I keep asking myself, 'Why do I have to do this?'"

Changes were made by the Getting Ready research staff between Rounds 1 and 2 in response to the ECPs' frustrations with aspects of both the individual and group coaching. Change was exemplified in an HS practitioner's comments on the group coaching during a Round 2 interview:

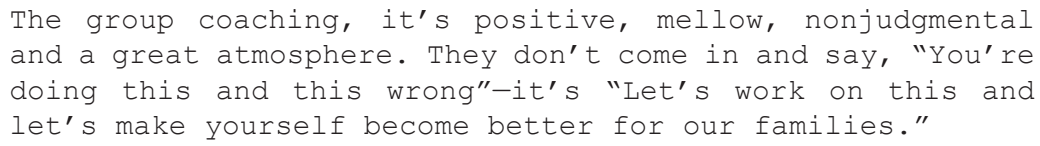

Most ECPs felt similar by the Round 2 interviews, at which time only five $(12 \%)$ reported any dissatisfaction with the group and individual

1. Modifications to the implementation of the professional development model occurred between Rounds 1 and 2 (Years 2 and 3 of the project). First, the delivery of coaching changed from a process in which two different coaches provided group and individual coaching to one in which a single coach provided both group and individual sessions for participants. This change was made in an effort to facilitate cohesion and integration across group and individual coaching, based on feedback from ECPs. Second, the amount of documentation for practitioners was reduced prior to Round 2 interviews based on feedback that demands were excessive. 
coaching. These coaching relationships endured, and ECPs wanted them to continue after the project ended.

The coaching staff was viewed as supportive by practitioners. Practitioners identified that group coaching support was important but that the structure of the support must be appropriate. Additionally, practitioners reported value in the debriefing of videotapes that occurred. The ongoing communication with ECPs and deliberate attempts by researchers to match identified needs with interpersonal preferences created new opportunities to foster effective experiences in coaching.

\section{Practice: Time Pressure and Paperwork Woes}

Despite the fact that most ECPs approached the intervention with hope, they also described challenges that were difficult to overcome. Participating in the Getting Ready intervention left some ECPs feeling overwhelmed, especially in Round 1. They often couched their discontent in praise for the objectives of the project but reported feeling that the increased paperwork resulted in unmanageable workloads. One practitioner within an SPP described how her initial interest was extinguished by time pressure and workload:

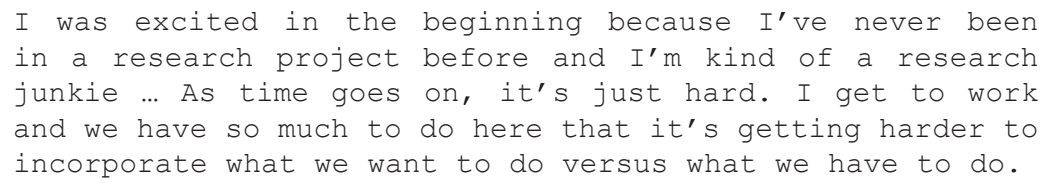

ECPs respected the goals and expectations of the parent engagement intervention, but when other job demands made meeting these expectations difficult, several reported that they would rather not participate. One teacher during Round 1 best described this dilemma by saying:

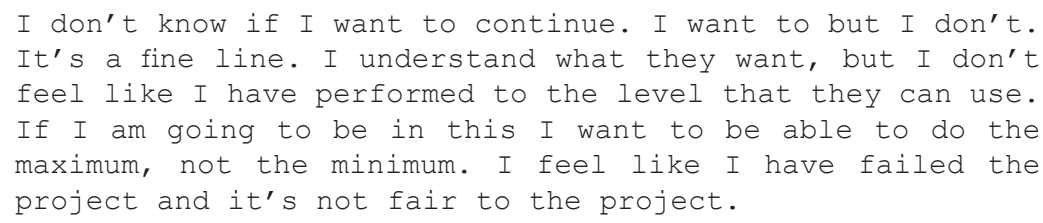

The Getting Ready project added more paperwork to the ECPs' workload. Seventeen (81\%) ECPs interviewed in Round 1 talked about the additional paperwork as a concern. One HS teacher described how the Getting Ready project had changed her job by disclosing, "It's gotten kind of crazy. I am bringing home stuff every night. What it has meant to me personally is more work, more paperwork." Another ECP stated a desire to "take the structure out, but leave the goals in." Although appreciating and connecting with the parent engagement goals of the project, this ECP felt overwhelmed with the demands of her job and the intervention's added expectations. 
Collectively, the ECPs' discontent with the paperwork was nonexistent in Round 2 interviews. Many ECPs commented that the Getting Ready research staff had listened to their concerns and made efforts to reduce the paperwork. This receptivity of the research staff was reported by 28 (70\%) of the ECPs and created a sense of respect and appreciation between the ECPs and the research team.

In sum, the interaction of research experiences and the realities of practice was expressed as an issue by many practitioners. ECPs were initially burdened by the amount of paperwork required by the intervention. The research team responded and made adjustments; responsiveness was viewed favorably by the participants and reduced discontent with the intervention.

\section{Discussion}

The purpose of this qualitative case study was to investigate the experiences of ECPs learning a new, conceptually unique intervention designed to support school readiness in children and families via parental engagement. It contributes to a growing literature base on effective professional development supports in early childhood (Gettinger \& Stoiber, 2008; National Professional Development Center on Inclusion, 2008; Pianta, 2006; Zaslow \& Martinez-Beck, 2006). Specifically, this investigation highlights the experiences and beliefs of practitioners involved in professional development designed to support and facilitate parent-child interactions (vs. curricular or programmatic changes). Understanding the experiences of ECPs is the next step in building a multidimensional understanding of professional development, particularly that focused on enhancing parental engagement. Applied, collaborative research that contributes to our understanding of professional development and related supports designed to impact high-quality programming for children and families is essential. The current investigation contributes to this charge.

In addition, this study builds on recent studies of the Getting Ready intervention that have indicated effective implementation as measured by behavioral indicators of ECPs' practices (Knoche et al., under review), generalization of the training philosophy to naturalistic teacher-parent communications (Edwards et al., under review), and positive effects of the parent engagement intervention on children and families (Cline et al., 2009; Sheridan et al., in press). These previous studies provide evidence that ECPs made visible changes in their behavior and that these changes influenced children and families. However, information on teachers' own experiences and beliefs within a conceptually unique parent engagement intervention, and perspectives of the professional development model that contributed to the observed and measured behavior changes, has not been available heretofore. Investigating the ECPs' perspectives on the parent engagement intervention, including professional development and coaching, 
provides information about their experiences regarding the new intervention, professional development, and ongoing supports intended to produce change. These observations may help us to understand how the ECPs shifted to a complex parent engagement model of practice. The reflections and feedback from ECPs collected via this qualitative case study approach provide rich evaluation data that inform researchers who are conducting collaborative research or attempting to institute a professional development approach to facilitating parental engagement.

The study indicates that (a) ECPs experienced the professional development as a spark that helped promote internalization of a new belief system and way of working and that ECPs were able to put parents much more at the center of their work, along with children, following the family-centered philosophy (Self-Perceived Changes in Confidence and Competence); (b) supportive relationships with coaches were felt by ECPs to be critical to the success of the parent engagement intervention (Relationships as Supports for Change); and (c) excessive reporting was encumbering, and lessening the paperwork load was an important part of the utility of professional development (Practice: Time Pressure and Paperwork Woes).

\section{Implications for Practice}

The thematic findings have implications for practice in early childhood education, especially in the context of research-based interventions intended to promote parental engagement. Taken together, the themes provide cross-cutting confirmation that (a) relationships are vital to the success of the implementation of a collaborative, research-based intervention; (b) opportunities for reflection and processing are an important component of the professional development model; (c) a long-term investment (more than one year) in the development of practitioners is important for a transformation of beliefs and perceived practices concerning work with families; and (d) a collaborative partnership with the research team is valuable for the practitioners.

Relationships are vital. The primary purpose of professional development in the Getting Ready intervention was to support ECPs in their development of competence and confidence in interactions with parents so as to support parents' own competence and confidence in their interactions with their children. As such, the professional development model was relationship based. Therefore, perhaps it is not surprising that trusting and productive coaching relationships were also critical to the practitioners' perceptions of the parent engagement intervention. When in a few situations the coaching relationship was felt to be lacking, commitment to the intervention study and willingness to be involved diminished. The research team had to be responsive to the needs of the ECPs to maximize participation. Identifying the mechanisms through which ECPs are most supported 
is critical to implementing successful professional development efforts in the context of collaborative research partnerships to facilitate parent engagement. The findings can be informative for other early childhood interventions that rely on buy-in from community practitioners.

Reflection and processing are important. As a function of both their involvement in a specific parent engagement training experience and the focus of the professional development elements on family-centered principles, the ECPs changed not only their reported practices but also their perceptions of families. ECP attitudes toward parental engagement in child learning and possibilities for working side by side with parents on behalf of children, in general, were positively affected by their participation and concomitant professional development opportunities. Given the content area of our intervention (i.e., parental engagement), this transformation represents a paradigm shift for many of the practitioners - from a focus on the child or parent to a focus on the relationship between the two. Although the study was not intended to focus on the mechanisms of the change process in ECPs, it is clear from their responses that they felt that opportunities for reflection and analysis such as those provided via coaching were important to promote growth and change in complex behavior. A positive change in practitioners' attitudes and philosophies toward their work with families may not have occurred had it not been for purposeful reflection (Hattie \& Timperley, 2007). These findings suggest that professional development efforts need to allow for sufficient opportunities for practitioners to ponder, discuss, and gradually assimilate the meaning of the changes expected of them.

Time is an important professional development investment. Along with the importance of relationships and reflection in the overall positive response to the intervention, we have learned that long-term investment is important in professional change. Ample time must be allowed when implementing a parent engagement intervention to ensure not only acquisition of skills but internalization of a philosophy of practice. Typically, professional development provides support for a single intervention year, with hopes of sustainability by practitioners over time. This time frame is likely dependent upon both the content of professional development and the form. We believe that this is especially true in situations where a change in an ECP's philosophy of practice (moving from child to parent focused) is required. Indeed, the change in responses from Round 1 to Round 2 for those who were reinterviewed indicates a movement from rote actions to deeper understandings and approaches to practice. Our study suggests that to effectively change professional behaviors related to strategies to support parental engagement, long-term resources need to be dedicated to professional supports. 
Collaboration is key to partnerships. In addition to time, professional development efforts must ensure sufficient opportunities for input and feedback; certainly this was the case with the Getting Ready project. The professional development approach used in this collaborative endeavor was dynamic in nature. Through the ongoing exchange among participants and researchers, attainment of the shared goals associated with family support, professional growth, and scientific investigation were more fully realized. Collaborative partnership in research benefits both researchers and practitioners in advancing implementation efforts of professional development approaches (Schensul, 1999) and is recommended practice (U.S. Department of Health and Human Services, 1990). Additionally, it is an encouraging finding that the culture of positive communication with colleagues and between research staff seemed to translate into more positive interactions with families in the project.

\section{Limitations}

The qualitative methods used in this case study allowed us to explore the ECPs' perceptions and understanding of the intervention over time; however, loss of ECPs from Round 1 to Round 2 was a limitation of the study. Seven new participants were recruited in Round 2. The loss of participants limited the possibility of making the focus of the study longitudinal changes over a 2-year period, but it did allow us to investigate the experiences of ECPs new to the intervention and to discover that they were echoing themes that were similar to those expressed by more experienced ECPs.

The case study method allowed us to explore perceptions and nuances of professional change from the individual experiences of ECPs, but it did not allow for systematic, experimental manipulation of variables; testing of different approaches to professional development (e.g., planned variations); or interpretation of objective results. Mechanisms by which change occurred over time, and causal effects of professional development practices, could not be determined. Given that other studies have shown that change in ECP behavior did occur, this study allows for exploration of critical internal processes that may have promoted the change process in ECPs. The findings suggest specific structures and experiences that may have facilitated the attainment of positive outcomes of the parent engagement intervention. For example, many of the ECPs consciously felt they got a spark for their work, felt support from coaches, saw visible change in parents, widened their focus from children to families and children, felt listened to by researchers, and internalized the philosophy of the parent engagement intervention.

\section{Conclusions}

The reflections by ECPs in this qualitative case study suggest the importance of researchers working in collaboration with practitioners to meet unique but mutually beneficial goals. Reflections by ECPs indicated that 
the ongoing assistance provided by research staff (coaches and consultants) provided support for the effective implementation of intervention efforts. Likewise, feedback provided by ECPs to researchers regarding needs, fit, and agency realities allowed for continual refinement of the professional development process. This mutual give-and-take ultimately contributed to a parent engagement intervention/professional development model that enabled effective parent-practitioner interactions, as reported by practitioners. ECP reports indicated that creating an environment that fostered communication between the ECPs and the research team and building relationships with coaches helped to create a culture of support that was meaningful to the practitioners and critical to their work with families. Future research should investigate whether this culture contributed to the success of the intervention and may be of particular value to others implementing professional development activities to support parental engagement within the context of large-scale community-research partnerships.

\section{Acknowledgments}

The development of this paper was supported by a grant awarded to Drs. Susan Sheridan and Carolyn Pope Edwards by the Department of Health and Human Services (DHHS) - National Institute of Child Health and Human Development (NICHD), Administration for Children and Families (ACF) and Office of the Assistant Secretary for Planning and Evaluation (ASPE); and the Department of Education (ED) - Office of Special Education and Rehabilitative Services. The opinions expressed herein are those of the investigators and do not reflect the funding agencies (Grant \#1R01H00436135). Appreciation is extended to the early childhood practitioners, coaches, and researchers who provided their time and insight to this project. The authors also thank Jessica Dempsey for her help with data analysis and coding.

\section{References}

Bellm, D., Burton, A., Whitebook, M., Broatch, L., \& Young, M. (2002). Inside the pre-K classroom: A study of staffing and stability in state-funded prekindergarten programs. Washington, DC: Center for the Childcare Workforce.

Bornstein, M. H., \& Tamis-LeMonda, C. S. (1989). Maternal responsiveness and cognitive development in children. New Directions for Child Development, 48, 49-61.

Bush Administration. (2002). Good start, grow smart. The Bush Administration's early childhood initiative. Accessed September 2, 2008 at http://www.whitehouse.gov/infocus/earlychildhood/toc.html

Christenson, S. L., \& Sheridan, S. M. (2001). Schools and families: Creating essential connections for learning. New York: Guilford Press.

Cline, K. D., Knoche, L. L., Edwards, C. P., Sheridan, S. M., \& Martinez, M. M. (2009, April). Getting Ready: One-year effects of a parent engagement on parenting behaviors in low-income families. Poster presented at the biennial meeting of the Society for Research in Child Development, Denver, CO.

Cohen, J. (1960). A coefficient for agreement for nominal scales. Education and Psychological Measurement, 20, 37-46. 
Creswell, J. W. (2003). Research design: Qualitative, quantitative, and mixed method research. Thousand Oaks, CA: Sage.

de Ruiter, C., \& van IJzendoorn, M. (1993). Attachment and cognition: A review of the literature. International Journal of Educational Research, 19, 5-20.

Dickinson, D., \& Brady, J. P. (2006). Toward effective support for language and literacy through professional development. In M. Zaslow \& I. Martinez-Beck (Eds.), Critical issues in early childhood professional development (pp. 141-170). Baltimore: Brookes.

Dowrick, P. W. (1994). A review of self modeling and related interventions. Applied and Preventive Psychology, 8, 22-39.

Fontaine, N. S., Torre, L. D., Grafwallner, R., \& Underhill, B. (2006). Increasing quality in early care and learning environments. Early Child Development and Care, 176, 157-169.

Foster, M. A., Lambert, R., Abbott-Shim, M., McCarty, F., \& Franze, S. (2005). A model of home learning environment and social risk factors in relation to children's emergent literacy and social outcomes. Early Childhood Research Quarterly, 20, 13-36.

Gettinger, M., \& Stoiber, K. (2008). Applying response-to-intervention model for emerging literacy development in low-income children. Topics in Early Childhood Special Education, 27, 198-213.

Ginsburg, H. P., Kaplan, R. G., Cannon, J., Cordero, M. I., Eisenband, J. G., Galanter, M., et al. (2006). Helping early childhood educators to teach mathematics. In M. J. Zaslow \& I. Martinez-Beck (Eds.),

Critical issues in early childhood professional development (pp. 171-202). Baltimore: Brookes.

Girolametto, L., Verbey, M., \& Tannock, R. (1994). Improving joint engagement in parentchild interaction: An intervention study. Journal of Early Intervention, 18, 155-167.

Hanft, B., Rush, D., \& Shelden, M. (2004). Coaching families and colleagues in early childhood. Baltimore: Brookes.

Harbin, G., Rous, B., \& McLean, M. (2005). Issues in designing state accountability systems. Journal of Early Intervention, 27, 137-164.

Hattie, J., \& Timperley, H. (2007). The power of feedback. Review of Educational Research, 77, $81-112$

Hill, N. E. (2001). Parenting and academic socialization as they relate to school readiness: The roles of ethnicity and family income. Journal of Educational Psychology, 93, 686-697.

Hosford, R. E. (1980). Self-as-a-model: A cognitive social learning technique. Counseling Psychologist, 9, 45-62.

Howes, C., James, J., \& Ritchie, S. (2003). Pathways to effective teaching. Early Childhood Research Quarterly, 18, 104-120.

Jackson, B., Larzelere, R., Clair, L. S., Corr, M., Fichter, C., \& Egertson, H. (2006). The impact of HeadsUp! Reading on early childhood educators' literacy practices and preschool children's literacy skills. Early Childhood Research Quarterly, 21, 213-226.

Justice, L. M., Mashburn, A. J., Hamre, B. K., \& Pianta, R. C. (2008). Quality of language and literacy instruction in preschool classrooms serving at-risk pupils. Early Childhood Research Quarterly, 23, 51-68.

Katz, L. G. (1992). What should young children be learning? Urbana, IL: ERIC Clearinghouse on Elementary and Early Childhood Education. (ERIC Document Reproduction Service No. ED290554)

Katz, L. G. (Ed.). (1995). Talks with teachers of young children: A collection. Norwood, NJ: Ablex.

Knoche, L. L., Sheridan, S. M., Edwards, C. P., \& Osborne, A. Q. (2008). Implementation of a relationship-based school readiness intervention: A multidimensional approach to fidelity measurement for early childhood. Manuscript submitted for publication.

Mahoney, G., \& Powell, A. (1988). Modifying parent-child interaction: Enhancing the development of handicapped children. Journal of Special Education, 22, 82-96.

Mashburn, A. J., \& Pianta, R. C. (2006). Social relationships and school readiness. Early Education and Development, 17, 151-176.

McCollum, J. A., Gooler, F., Yates, T. J., \& Appl, D. (2001). PIWI: Enhancing parent-child interaction as a foundation for early intervention. Infants and Young Children, 14, 34-45. 
McCollum, J. A., \& Yates, T. J. (1994). Dyad as focus, triad as means: A family-centered approach to supporting parent-child interactions. Infants and Young Children, 6, 54-63.

McWayne, C., Hampton, V., Fantuzzo, J., Cohen, H., \& Sekino, Y. (2004). A multivariate examination of parent involvement and the social and academic competencies of urban kindergarten children. Psychology in the Schools, 41, 1-15.

National Professional Development Center on Inclusion. (2008). What do we mean by professional development in the early childhood field? Chapel Hill: The University of North Carolina, FPG Child Development Institute. Accessed at http://www.fpg.unc.edu/ npdci

No Child Left Behind Act of 2001, Pub. L. No. 107-110, 115 Stat. 1425 (2002).

Pianta, R. (2006). Standardized observation and professional development: A focus on individualized implementation and practices. In M. Zaslow \& I. Martinez-Beck (Eds.), Critical issues in early childhood professional development (pp. 231-254). Baltimore: Brookes.

Raver, C. C., \& Knitzer, J. (2002). Ready to enter: What research tells policymakers about strategies to promote social and emotional school readiness among three-and four-year-old children. New York: National Center for Children in Poverty.

Schensul, J. (1999). Organizing community research partnerships in the struggle against AIDS. Health Education and Behavior, 26, 266-283.

Sheridan, S. M., \& Edwards, C. P. (2003). Parent engagement and child learning birth to five. Lincoln: University of Nebraska-Lincoln; National Institute of Child Health and Human Development, Administration for Children and Families and Office of the Assistant Secretary for Planning and Evaluation; and the Department of Education, Office of Special Education and Rehabilitative Services.

Sheridan, S. M., Knoche, L. L., Edwards, C. P., Bovaird, J. A., \& Kupzyk, K. A. (in press). Parent engagement and school readiness: Effects of the Getting Ready intervention on preschool children's social-emotional competencies and behavioral concerns. Early Education and Development.

Sheridan, S. M., \& Kratochwill, T. R. (1992). Behavioral parent-teacher consultation: Conceptual and research considerations. Journal of School Psychology, 30, 117-139.

Sheridan, S. M., \& Kratochwill, T. R. (2008). Conjoint behavioral consultation: Promoting familyschool connections and interventions. New York: Springer.

Sheridan, S. M., Marvin, C. A., Knoche, L. L., \& Edwards, C. P. (2008). Getting Ready: Promoting school readiness through a relationship-based partnership model. Early Childhood Services: An Interdisciplinary Journal of Effectiveness, 2, 149-172.

Shonkoff, J. P., \& Phillips, D. (2000). From neurons to neighborhoods. Washington, DC: National Academy Press.

Snow, K. L. (2006). Measuring school readiness: Conceptual and practical considerations. Early Education and Development, 17, 7-41.

Stake, R. E. (1995). The art of case study research. Thousand Oaks, CA: Sage.

Strauss, A., \& Corbin, J. (1990). Basics of qualitative research: Grounded theory procedures and techniques. London: Sage.

Thompson, R. A. (2002). The roots of school readiness in social and emotional development. Kauffman Early Education Exchange, 1, 8-29.

Turner, L. A., \& Burke, J. (2003). A model of mastery motivation for at-risk preschoolers. Journal of Educational Psychology, 95, 495-505.

U.S. Department of Health and Human Services. (1990). Head Start research and evaluation: A blueprint for the future (DHHS Publication No. ACY 91-31195). Washington, DC: Author.

Weigel, D. J., Martin, S. S., \& Bennett, K. K. (2006). Contributions of the home literacy environment to preschool-aged children's emerging literacy and language skills. Early Child Development and Care, 176, 357-378.

Yin, R. (1994). Case study research: Design and methods (2nd ed.). Beverly Hills, CA: Sage.

Zaslow, M., \& Martinez-Beck, I. (Eds.). (2006). Critical issues in early childhood professional development. Baltimore: Brookes. 